\title{
MELHORAMENTO GENETTICO DA CANA-DE-AÇÚCAR: VII. ENSAIOS DE CLONES IAC, SÉRIE 1977, EM LATOSSOLO VERMELHO-ESCURO, NA REGIÃO DE RIBEIRÃo PRETO (SP) $\left(^{1}\right.$ )
}

\author{
MARCOS GUIMARÃES DE ANDRADE LANDELL $\left({ }^{2,5}\right)$, RAPHAEL ALVAREZ $\left({ }^{3}\right)$, \\ JOSÉ CARLOS VILA NOVA ALVES PEREIRA $\left({ }^{2}\right)$ \\ e MARIA BERNADETE SILVAROLLA
}

\begin{abstract}
RESUMO
Com a finalidade de estudar 16 clones de cana-de-açúcar, provenientes de hibridaçð̄es efetuadas em Camamu (BA) em 1977, efetuou-se um ensaio em latossolo vermelho-escuro, na Usina São Martinho, Pradópolis (SP). No ensaio, plantado em março de 1986, utilizou-se o delineamento em blocos ao acaso, com quatro repetições, sendo a análise estatística feita com a média de quatro colheitas. Avaliaram-se as produtividades de cana e açúcar pol (\%) cana, fibra (\%) cana, populaçăo de colmos e intensidade de florescimento. Considerando-se essas características, assim como a reação ao carvão e tomando-se como padrőes as variedades SP70-1143, IAC64-257, NA56-79 e IAC58-480, revelaram-se os clones IAC77-186, IAC77-51 e IAC77-192, como novas opçōes para a composição de estudos de manejo varietal em condiçōes edafociimáticas similares.
\end{abstract}

Termos de indexação: cana-de-açúcar, Saccharum spp., melhoramento, hibridação, avaliação de clones.

\author{
ABSTRACT \\ SUGARCANE BREEDING: VII. EVALUATION OF SERIES 1977 IAC \\ SUGARCANE CLONES, IN DARK RED LATOSOLS, IN RIBEIRĀO PRETO \\ REGION, STATE OF SÃO PAULO, BRAZIL
}

In order to study 16 sugarcane clones, obtained from hybridizations made in 1977, in Camamu, State of Bahia, it was carried out an experiment in Dark Red Latosols, at São Martinho Mill, Pradópolis, State of São Paulo, field trial was started in 1986, using a randomized complete block design with four replications. Statistical analysis was performed with four harvest means. It was evaluated cane and sugar yield ( $\mathrm{t} / \mathrm{ha}$ ), sugar content (pol \% cane), fiber \% cane, number of stalks and flowering. According to these characteristics, in addition to the level of resistance to smut, studied comparatively to four varieties used as control, SP70-1143, IAC64-257, NA56-79 and IAC58-480, the clones IAC77-186, IAC77-51 and IAC77-192 showed to be new options to be studied for varietal management in similar environmental conditions.

Index terms: sugarcane, Saccharum spp., breeding, hybridization, clone evaluation.

\footnotetext{
( 1 ) Recebido para publicação em 29 de janeiro e aceito em 2 de setembro de 1992.

$\left(^{2}\right)$ Estação Experimental de Ribeirão Preto, Instituto Agronômico de Campinas (IAC), Caixa Postal 171, 14001-970 Ribeirão Preto (SP).

(3) Seção de Cana-de-Açúcar, IAC, Caixa Postal 28, 13001-970 Campinas (SP).

() Seção de Genética, IAC.

(5) Com bolsa de pesquisa do CNPq.
} 


\section{INTRODUÇÃO}

Os estudos desenvolvidos pelo Instituto Agronômico de Campinas, em cana-de-açúcar, forneceram importantes subsídios técnicos para a implantação e posterior expansão da cultura no Estado de São Paulo (Segalla \& Alvarez, 1964, 1968). Dessa forma, em 1983, cerca de $95 \%$ da área paulista plantada com cana constava de variedades estudadas e, posteriormente, recomendadas pelo programa do Instituto Agronômico, incluindo-se, aí, aquelas criadas sob a sigla IAC e/ou introduzidas de outros centros de melhoramento (Alvarez et al., 1982, 1983, 1984, 1986; Segalla \& Alvarez, 1956, 1958; Segalla et al., 1980, 1982).

A partir de 1983, visando à ampliação dos trabalhos de melhoramento, bem como ao estudo mais efetivo da interação do genótipo com o meio ambiente, e contando com a colaboração de pesquisadores das Estaçōes Experimentais do IAC, iniciou-se a implantação de Centros Regionais de Melhoramento da Cana-de-Açúcar, em Sāo Paulo, definindo-se, a princípio, como regiōes representativas, Piracicaba, Ribeirão Preto, Jaú, Mococa, Pindorama, Assis, Adamantina e Votuporanga $\left({ }^{6}\right)$.

O presente trabalho refere-se aos estudos desenvolvidos na região de Ribeirão Preto, com clones IAC originários de hibridaçōes realizadas cm 1977. Para tanto, os clones foram avaliados quanto a parâmetros de produtividade agroindustrial, comparativamente a variedades comerciais, utilizadas como padrāo.

\section{MATERIAL E MÉTODOS}

O experimento foi realizado na Usina São Martinho, em Pradópolis (SP), em latossolo vermelho-escuro, textura argilosa, cujas características químicas são apresentadas no quadro 1.

O delineamento experimental foi o de blocos casualizados, com quatro repetiçōes, sendo avaliados 16 clones IAC, série 1977 , em relação às variedades NA56-79, SP70-1143, IAC58-480 e IAC64-257, consideradas, na ocasião, como padrões varietais. Os clones IAC avaliados, assim como seus progenitores, foram os seguintes:

6) R. ALVAREZ. Aspectos da pesquisa com cana-de-açúcar no Instituto Agronômico, com ênfase ao melhoramento genético. Palestra apresentada no auditório do Instituto Agronômico de Campinas, em 27 de março de 1990.
CLONES

IAC77-10

IAC77-36

IAC77-51

IAC77-53

IAC77-81

AC77-90

IAC77-92

IAC77-112

IAC77-113

IAC77-153

IAC77-165

IAC77-175

IAC77-186

IAC77-192

IAC77-205
IAC77-173
PROGENITORES

IAC58-480 X IAC52-150

IAC58-480 X IAC48-65 •

IAC58-480 X NA56-79

IAC58-480 X NA56-79

IAC58-480 X NA56-79

IAC58-480 X NA56-79

IAC58-480 X NA56-79

LAC58-480 X NA56-79

IAC58-480 X NA56-79

TROJAN X Co775

IAC58-480 PC

IAC58-480 PC

IAC58-480 PC

IAC58-480 PC

IAC58-480 PC

IAC51-201 PC
As parcelas eram constituídas de três linhas de $10 \mathrm{~m}$, espaçadas de $1,50 \mathrm{~m}$ entre si, perfazendo a área útil de $45 \mathrm{~m}^{2}$. As adubações utilizadas na cana-planta e nas socas foram feitas segundo a análise química do solo (Raij et al., 1985) e a calagem segundo o método de saturação por bases, visando à elevação do $\mathrm{V}$ a $60 \%$.

Efetuou-se o plantio em 25 de março de 1986 e, as colheitas, em 21-8-87, 26-8-88, 14-8-89 e 10-8-90, para cana-planta e socas respectivamente. Nessas ocasiōes, amostraram-se dez colmos seguidos na linha, em cada parcela, para a análise tecnológica (Tanimoto, 1964).

A queda de produção agrícola no $2^{\circ}, 3^{\circ}$ e $4^{\circ}$ cortes foi obtida empregando-se a expressão $\mathrm{Qx} \%$ $=\left[\left(\mathbf{P}_{1}-\mathbf{P}_{\mathbf{x}}\right) \div \mathbf{P}_{1}\right] .100$, adaptada de Alvarez et al. (1987), onde $P_{x}$ representa a produção do corte considerado e $P_{1}$, a produção da cana-planta.

Nos dados referentes ao florescimento, atribuíram-se as seguintes notas: 0 , clones cujos colmos nāo apresentassem florescimento; 1 , clones com menos de $10 \%$ de colmos florescidos; 2 , quando 10 a $20 \%$ dos colmos estivessem florescidos e, 3, quando o florescimento atingisse mais de $20 \%$ dos colmos.

Os dados referentes à reação ao agente causador do carvão-da-cana (Ustilago scitaminea 
Quadro 1. Características químicas do latossolo vermelho-escuro, textura argilosa, da Usina São Martinho, Pradópolis (SP)

\begin{tabular}{|c|c|c|c|c|c|c|c|c|c|c|c|c|}
\hline $\begin{array}{l}\text { Profun- } \\
\text { didade }\end{array}$ & M.O. & $\mathbf{P}$ & $\mathrm{K}^{+}$ & $\mathrm{Ca}^{2+}$ & $\mathrm{Mg}^{2+}$ & $\mathbf{H}+\mathbf{A l}$ & $\mathrm{Al}^{3+}$ & $S$ & CTC & $\mathbf{M}$ & V & $\begin{array}{c}\mathrm{pH} \\
\mathrm{CaCl}_{2}\end{array}$ \\
\hline $\mathrm{cm}$ & $\%$ & ppm & $\ldots$ & $L_{\mathbf{m}}$ & $0 \mathrm{~cm}^{3}$ & 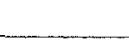 & & & & $\%$ & $\%$ & \\
\hline 0.25 & 1,94 & 27 & 0,10 & 1,18 & 0,42 & 3,43 & 0,07 & 33,14 & 5,13 & 3,95 & 15,10 & 4,80 \\
\hline $25-50$ & 1,68 & 13 & 0,10 & 0,91 & 0,34 & 3,43 & 0,17 & 28,24 & 4,78 & 11,18 & 14,21 & 4,60 \\
\hline
\end{tabular}

Sydow) foram fornecidos pela Comissão de Controle do Carvão da Cana-de-Açúcar do Estado de São Paulo, que se utilizou do método descrito em Figueiredo et al. (1980).

Realizaram-se análises estatísticas dos dados de produtividade de cana ( $\mathrm{t}$ de colmos/ha), teor de açúcar (pol \% cana), produtividade de açúcar ( $\mathrm{t}$ de $\mathrm{pol} / \mathrm{ha}$ ) e fibra \% cana, utilizando-se, para as comparaçōes de médias, o teste de Tukey a $5 \%$.

No decorrer do experimento, foram efetuadas observações relativas ao período de maturação para cada material em estudo, cujos dados não foram apresentados. Calculou-se, ainda, a produtividade de cana/hectare/dia ( $t /$ ha/dia), resultante da razão entre a produtividade de cana ( $t / h a)$ e o número total de dias transcorridos em cada ciclo. Somente para o quarto corte, avaliou-se o número de colmos/hectare, a partir da determinação do número total de colmos verificados em cada unidade experimental, bem como o peso médio de colmo, como resultado da razão entre o peso total da parcela e o número total de colmos por ela produzidos.

Os dados de precipitação pluvial e de temperaturas máximas e mínimas, registrados no período março de 1986-agosto de 1990, em Pradópolis, foram reunidos nas figuras 1 e 2 respectivamente.

\section{RESULTADOS E DISCUSSÃO}

O quadro 2 apresenta os valores médios de produtividade de cana, teor de sacarose, produtividade de açúcar e fibra referentes aos quatro cortes, bem como dados relativos ao florescimento. Os valores de produtividade de cana/dia, número e peso médio de colmos no $4^{\circ}$. corte, os índices de queda de produção ao longo das colheitas e reação ao carvão foram reunidos no quadro 3 .

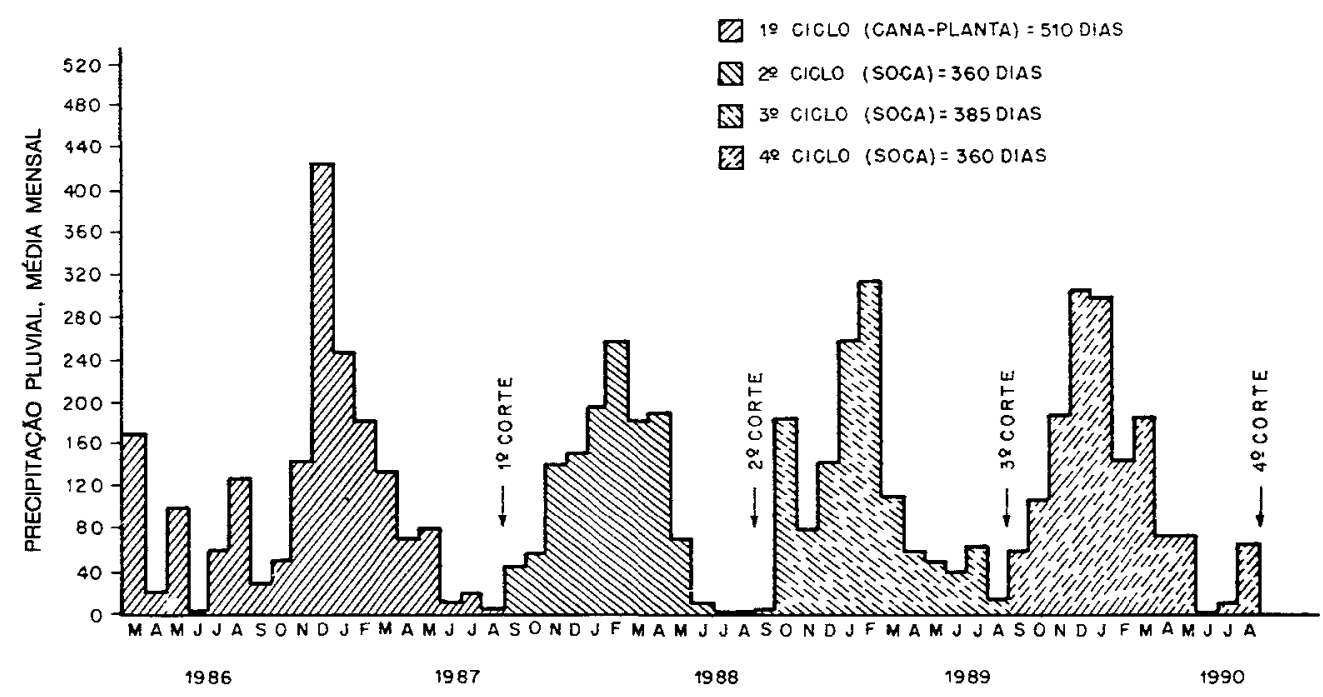

Figura 1. Dađos de precipitação pluvial (média mensal em mm) registrađos no período março/86-agosto/90 em Prađópolis (SP) 


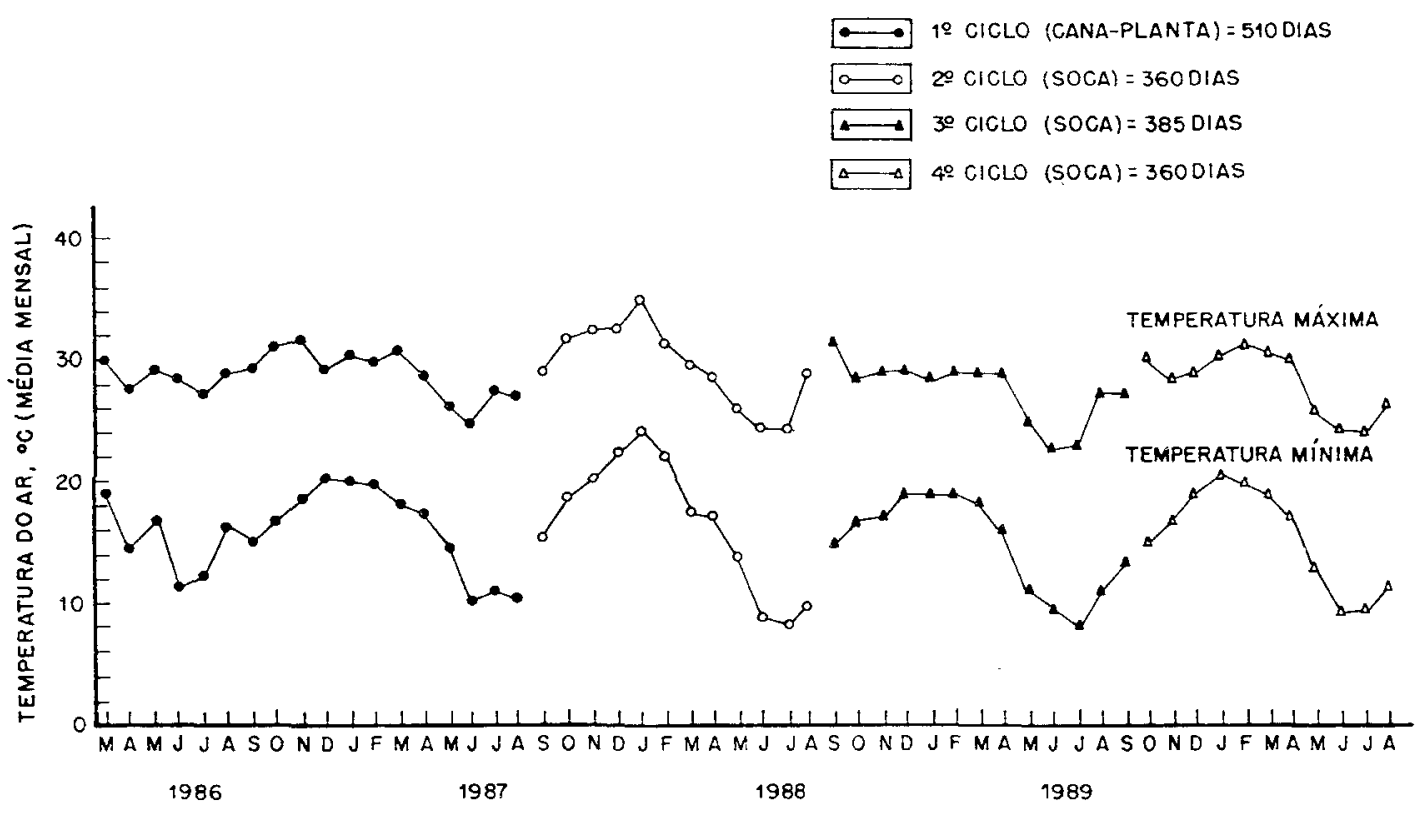

Figura 2. Dados de temperaturas máximas e mínimas (médias mensais em ${ }^{\circ} \mathrm{C}$ ) registrados no periodo março/86-agosto/90 em Pradópolis (SP).

Com relação à produtividade de cana, destacaram-se os clones IAC77-186 IAC77-51 e IAC77-192, que não diferiram estatisticamente do melhor padrão, SP70-1143, apresentando valores absolutos próximos aos da NA56-79. Os clones IAC77-53, IAC77-165 e IAC77-90 equivaleram-se estatisticamente aos padrões IAC64-257 e NA56-79.

Sobressaíram-se, quanto ao teor de sacarose, dentre aqueles de maior potencial agrícola, os clones IAC77-186, IAC77-53 e IAC77-165, que não diferiram estatisticamente dos clones IAC77-153 e IAC77-92 e dos padrōes SP70-1143 e NA56-79, que foram os melhores para essa característica.

Considerando-se de forma conjunta os componentes de produtividade mencionados, destacaram-se os clones IAC77-186, IAC77-51, IAC77-53, IAC77-165 e IAC77-192, cujos valores de TPH não diferiram estatisticamente dos padrões.
O quadro 3 revela que, entre os melhores clones em produçāo de açúcar por área, o IAC77-186 foi o genótipo com os menores índices de queda de produtividade ao longo das colheitas. Com baixo teor de fibra e bom peso médio do colmo nas socas, mostrou maior teor de sacarose no período de final de safra (conforme Camargo et al., 1982). Isso, aliado à ausência de florescimento e à resistência ao carvão, permite sua utilização naquele período.

O IAC77-51 aproximou-se da variedade IAC64-257 no que diz respeito à produtividade de cana/dia, ao teor de fibra, à população de colmo, ao peso médio do colmo e à manutenção da produtividade agrícola ao longo dos cortes. Diferenciou-se, porém, desse padrão, quanto ao período de máximo teor de sacarose que permite sua utilização no período compreendido entre junho e meados de setembro (dados não publicados), além de demonstrar resistência ao carvão da cana. 
Quadro 2. Valores médios (4 cortes) de produtividade de cana e açúcar, teor de sacarose, fibra (\%) cana, intensidade de florescimento e resumo das análises estatísticas dos dados do experimento com clones IAC, série 1977, em latossolo vermelho-escuro, na Usina São Martinho, Pradópolis (SP)

\begin{tabular}{|c|c|c|c|c|c|}
\hline Tratamentos & $\begin{array}{l}\text { Produtividade } \\
\text { de cana }\end{array}$ & $\begin{array}{l}\text { Teor de } \\
\text { sacarose }\end{array}$ & $\begin{array}{l}\text { Produtividade } \\
\text { de açúcar }\end{array}$ & Fibra & Florescimento $\left({ }^{1}\right)$ \\
\hline & t/ha & pol \% cana & $\mathrm{t}$ de $\mathrm{pol} / \mathrm{ha}$ & $\%$ & \\
\hline SP70-1143 & $130,3 a$ & $15,57 a-d$ & $20,32 a$ & $13,28 \mathrm{ab}$ & 1 \\
\hline NA56-79 & $116,7 a-c$ & $16,32 \mathrm{ab}$ & $19,14 a b$ & $12,69 b c$ & 1 \\
\hline IA64-257 & $122,4 a b$ & $15,39 b-d$ & $18,92 \mathrm{ab}$ & $12,43 \mathrm{bc}$ & 0 \\
\hline LAC77-186 & $116,1 a-c$ & $15,95 a-c$ & $18,61 \mathrm{a}-\mathrm{c}$ & $10,24 d$ & 0 \\
\hline IAC77-51 & $116,0 \mathrm{a}-\mathrm{c}$ & $15,39 b-d$ & $18,00 a-d$ & $12,19 b c$ & 0 \\
\hline IAC77-53 & $109,5 b-e$ & $16,27 \mathrm{ab}$ & $17,79 a-d$ & $13,08 b c$ & 1 \\
\hline IAC77-165 & $109,1 b-e$ & $16,20 \mathrm{ab}$ & $17,78 a-d$ & $12,17 \mathrm{bc}$ & 0 \\
\hline IAC77-192 & $115,2 a-d$ & $15,18 b-d$ & $17,59 \mathrm{ab}$ & $13,25 \mathrm{ab}$ & 0 \\
\hline IAC77-153 & $99,8 c-f$ & $16,70 \mathrm{a}$ & $16,69 b-d$ & $12,92 b c$ & 1 \\
\hline LAC77-90 & $103,1 b-f$ & $15,69 a-d$ & $16,23 b-d$ & $11,95 b c$ & 0 \\
\hline IAC77-205 & $99,4 c-f$ & $16,17 \mathrm{ab}$ & $16,10 \mathrm{~b}-\mathrm{d}$ & $13,14 a-c$ & 0 \\
\hline IAC77-173 & $99,8 c-f$ & $15,79 a-d$ & $15,99 \mathrm{~b}-\mathrm{e}$ & $11,52 c-d$ & 0 \\
\hline IAC58-480 & $97,3 c-f$ & $16,23 \mathrm{ab}$ & $15,89 \mathrm{~b}-\mathrm{e}$ & $12,37 \mathrm{bc}$ & 0 \\
\hline IAC77-112 & $99,2 c-f$ & $15,36 b-d$ & $15,30 c-f$ & $13,40 \mathrm{ab}$ & 0 \\
\hline IAC77-10 & $96,4 c-f$ & $15,64 a-d$ & $15,12 \mathrm{~d}-\mathrm{f}$ & $12,62 b c$ & 0 \\
\hline IAC77-92 & $89,8 \mathrm{e}-\mathrm{g}$ & $16,57 \mathrm{a}$ & $14,90 \mathrm{~d}-\mathrm{f}$ & $11,93 b-d$ & 1 \\
\hline IAC77-36 & $94,9 d-f$ & $15,36 b-d$ & $14,66 \mathrm{~d}-\mathrm{f}$ & $14,82 \mathrm{a}$ & 3 \\
\hline IAC77-81 & $85,7 \mathrm{f}-\mathrm{g}$ & $14,67 d$ & $12,73 \mathrm{e}-\mathrm{g}$ & $13,35 \mathrm{ab}$ & 1 \\
\hline IAC77-113 & $83,4 \mathrm{f}-\mathrm{g}$ & $14,81 \mathrm{~cd}$ & $12,09 \mathrm{fg}$ & $13,61 \mathrm{ab}$ & 0 \\
\hline IAC77-175 & $69,7 \mathrm{~g}$ & $15,58 \mathrm{a}-\mathrm{d}$ & $10,81 \mathrm{~g}$ & $12,25 b c$ & 0 \\
\hline F (trat.) & $14,02 * *$ & $6,32^{* *}$ & $14,56^{* *}$ & $8,45^{* *}$ & \\
\hline d.m.s. (Tukey 5\%) & 20,46 & 1,16 & 3,37 & 1,70 & \\
\hline C.V.\% & 7,58 & 2,80 & 7,89 & 5,11 & \\
\hline
\end{tabular}

$\left({ }^{1}\right)$ Escala de 0 a 3 pontos: $0=$ sem florescimente; $1=<10 \%$ de florescimento; $2=10-20 \%$ de florescimento, e $3=>20 \%$ de florescimento.

O IAC77-192 apresentava, até o 3. corte, produtividade agrícola superior aos demais clones em teste. No entanto, a acentuada queda de produção, no $4^{\circ}$. corte, comprometeu sua produtividade média. No mais, equivaleu-se ao IAC77-51, com exceção do seu período de colheita, que abrange agosto a novembro. Destaca-se ainda que, de acordo com observaçōes preliminares feitas na Usina São Martinho, em solos com fertilidade superior ao do utilizado para este ensaio, com irrigação suplementar, esse clone mostrou produtividades de cana e de açúcar equivalentes às das variedades SP70-1143 e NA56-79.

O IAC77-53 tem período de colheita semelhante ao IAC77-51; apresenta, no entanto, os inconvenientes do florescimento e da isoporizaçāo, bem como da diminuição acentuada do peso médio dos colmos nas socas. 
Quadro 3. Valores de produtividade de cana/ha/dia, número de colmos e peso médio dos colmos no $4^{2}$ corte, índices de queda de produção na $2^{a}, 3^{a}$ e $4^{a}$ colheitas e reação ao carvão em ensaio na Usina São Martinho, Pradópolis, em latossolo vermelho-èscuro, com clones IAC, série 1977

\begin{tabular}{|c|c|c|c|c|c|c|c|c|c|}
\hline \multirow{2}{*}{ Tratamentos } & \multicolumn{4}{|c|}{ Produção de cana/ha/dia } & \multicolumn{3}{|c|}{ Índices de queda } & \multirow{2}{*}{$\begin{array}{l}\text { Colmos/ha } \\
\text { (4ㅇ corte) }\end{array}$} & \multirow{2}{*}{$\begin{array}{l}\text { Peso Reação ao } \\
\text { médio carvāo }\left(^{1}\right)\end{array}$} \\
\hline & $1^{9}$ ciclo & $2^{\circ}$ ciclo & $3^{\circ}$ ciclo & $4^{\circ}$ ciclo & Q2 & Q3 & Q4 & & \\
\hline
\end{tabular}

\begin{tabular}{|c|c|c|c|c|c|c|c|c|c|c|}
\hline & & & & & & & & & & \\
\hline & & t & & - & 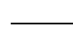 & $-\%$ & _ & $\mathrm{n}^{\mathrm{o}}$ & $\mathbf{k g}$ & \\
\hline IAC77-10 & 0,25 & 0,27 & 0,23 & 0,21 & 22,6 & 28,0 & 41,0 & 83.511 & 0,82 & MS \\
\hline IAC $77-36$ & 0,24 & 0,27 & 0,22 & 0,21 & 20,4 & 29,9 & 41,1 & 121.733 & 0,56 & $\mathbf{R}$ \\
\hline IACT7-51 & 0,29 & 0,33 & 0,27 & 0,27 & 19,8 & 30,0 & 33,8 & 103.222 & 0,88 & $\mathbf{R}$ \\
\hline LAC $77-81$ & 0,22 & 0,26 & 0,21 & 0,16 & 14,6 & 25,8 & 47,7 & 68.289 & 0,79 & MS \\
\hline IAC77-90 & 0,24 & 0,29 & 0,27 & 0,22 & 17,0 & 15,2 & 35,4 & 97.000 & 0,77 & $\mathbf{R}$ \\
\hline IAC $77-92$ & 0,20 & 0,26 & 0,23 & 0,21 & 8,8 & 15,7 & 28,4 & 84.889 & 0,81 & $\mathbf{R}$ \\
\hline IAC77-153 & 0,29 & 0,24 & 0,24 & 0,19 & 40,8 & 37,1 & 52,5 & 95.156 & 0,69 & MS \\
\hline IAC77-165 & 0,31 & 0,29 & 0,27 & 0,20 & 33,1 & 34,3 & 54,3 & 75.444 & 0,89 & MS \\
\hline IAC77-173 & 0,28 & 0,28 & 0,23 & 0,19 & 30,4 & 38,7 & 52,2 & 81.622 & 0,78 & $\mathbf{R}$ \\
\hline IAC $77-175$ & 0,22 & 0,18 & 0,16 & 0,11 & 43,9 & 46,9 & 66,4 & 44.178 & 0,82 & $\mathbf{S}$ \\
\hline IAC77-186 & 0,26 & 0,34 & 0,29 & 0,27 & 10,0 & 18,4 & 28,9 & 92.178 & 0,98 & $\mathbf{R}$ \\
\hline IAC77-192 & 0,29 & 0,34 & 0,28 & 0,24 & 17,3 & 27,9 & 41,7 & 99.044 & 0,81 & $\mathbf{R}$ \\
\hline NAS6-79 & 0,29 & 0,33 & 0,30 & 0,24 & 19,8 & 23,6 & 42,7 & 87.444 & 0,91 & MS \\
\hline
\end{tabular}

(1) R: resistente; S: suscetível e MS: moderadamente suscetível.

A sensível queda de produçāo agrícola nas socas, causada principalmente pela diminuição na população de colmos, aliada à suscetibilidade ao carvão, tornaram o IAC77-165 inviável como cultivar.

\section{CONCLUSŌES}

De acordo com o conjunto das características agroindustriais analisadas, os clones IAC77-51, IAC77-186 e IAC77-192 apresentaram condiçōes de ser incluídos em estudos de manejo varietal, para a região de Ribeirão Preto (SP).

\section{AGRADECIMENTOS}

Aos Engenheiros-Agrônomos Sérgio Bicudo Paranhos, Júlio Marcos Campanhão e José Cristovão Momesso, da Usina São Martinho, e aos Técnicos Agrícolas Joāo Carlos Taveira Neves e José Osmar Barbosa. Especial deferência à Copersucar, por per- mitir a utilização de seus campos de cruzamento na Estação Experimental de Camamu (BA).

\section{REFERÊNCIAS BIBLIOGRÁFICAS}

ALVAREZ, R.; BASTOS, C.R.; LANDELL, M.G. de A.; BOVI, V.; POMMER, C.V.; PEREIRA, J.C.V.N.A.; SILVAROLLA, M.B.; GODOY JÚNIOR, G.; COSTA, A.A.; CAMARGO, A.P. de \& RAMOS, M.T.B. Melhoramento da cana-de-açúcar: V. Experimentos regionais com clones obtidos em 1970. STAB, Piracicaba, 4(4):39-46, 1986.

ALVAREZ, R.; BASTOS, C.R.; POMMER, C.V.; BRINHOLI, O.; DALBEN, A.E.; GODOY JÚNIOR, G.; BOVI, V. \& CIONE, J. Melhoramento da cana-de-açúcar: III. Experimentos regionais com clones obtidos em 1968. In: CONGRESSO NACIONAL DA SOCIEDADE DE TÉCNICOS AÇUCAREIROS DO BRASIL, 2., e SIMPÓSIO LATINO-AMERICANO SOBRE MODALIDADES DE FINANCIAMENTO À PRODU. ÇÃO DE ENERGIA RENOVÁVEL, 1., Rio đe Janeiro, 1981. Anais. Rio de Janeiro, STAB, 1981. v.2, p.198-215. 
ALVAREZ, R; BASTOS, C.R.; SEGALLA, A.L.; OLIVEIRA, H. de; GODOY JÚNIOR, G.; POMMER, C.V.; BRINHOLI, O. \& DALBEN, A.E. Melhoramento da cana-de-açúcar. Ila. Experimentos regionais com clones obtidos em 1967. Bragantia, Campinas, 42:27-36, 1983.

ALVAREZ, R.; POMMER, C.V.; BASTOS, C.R.; BRINHOLI, O.; GODOY JÚNIOR, G. \& BOVI, V. Melhoramento da cana-deaçúcar: IV. Experimentos regionais com clones obtidos em 1969. Bragantia, Campinas, 43(1):155-165, 1984.

ALVAREZ, R.; SEGALLA, A.L.; LANDELL, M.G. de A.; SILVAROLLA, M.B. \& GODOY JÚNIOR, G. Melhoramento genético da cana-de-açúcar: avaliação de clones provenientes de hibridações efetuadas em 1965. Bragantia, Campinas, 46(1):121-126, 1987.

CAMARGO, A.P. de; BOVI, V.; ALVAREZ, R.; GODOY JÚNIOR, G.; POMMER, C.V.; LANDELL, M.G. de A.; ZIMBACK, L.; IGUE, T. \& SILVA, M.T.R. da. Melhoramento genético da cana-de-açúcar: avaliaçāo de clones provenientes de hibridaçס̄es realizadas em 1974, 1977, 1978 e 1979. Bragantia, Campinas, 51(1):39-47, 1992.

FIGUEIREDO,F.; MATSUOKA, S.; SILVA, W.M. da;TOKESHI, H.; FIGUEIREDO JÚNIOR, E.R.; SILVEIRA, A.P. da; MELLO, S.S. \& PARADELA FILHO, O. Testes de resisténcia de variedades de cana-de-açúcar a Ustilago scitaminea Syd. em condições de campo. O Biológico, São Paulo, 46(10):225-233, 1980.
RAIJ, B. van; SILVA, N.M. da; BATAGLIA, O.C; QUAGGIO, J.A.; HIROCE, R.; CANTARELLA, H.; BELLINAZZI JÚNIOR, R; DECHEN, A.R. \& TRANI, P.E. Recomendaçōes de adubação e calagem para o Estado de Sáo Paulo. Campinas, Instituto Agronômico, 1985. 107p. (Boletim técnico, 100)

SEGALLA, A.L \& ALVAREZ, R. Ensaios de variedades de cana-de-açúcar: I. Série de ensaios realizados noperiodo de 1951 a 1954. Bragantia, Campinas, 15:373-392, 1956.

SEGALLA, A.L. \& ALVAREZ, R. Variedades de cana-de-açúcar. II. Série de ensaios realizados no período de 1953-1956. Bragantia, Campinas, 17:45-79, 1958.

SEGALLA, A.L. \& ALVAREZ, R. Melhoramento da cana-deaçúcar: I. Experiências com "seedlings" obtidos em 1947, 1948 e 1949. Bragantia, Campinas, 23:187-223, 1964.

SEGALLA, A.L. \& ALVAREZ, R. Contribuição ao desenvolvimento da cultura da cana-de-açúcar em São Paulo. O Agronômico, Campinas, 20(5/6):1-35, 1968.

SEGALLA, A.L.; ALVAREZ, R.; OLIVEIRA, H. de; IGUE, T. \& GODOY JÚNIOR, G. Melhoramento da cana-de-açúcar: II. Experimentos regionais com clones obtidos em 1964. Bragantia, Campinas, 41:109-117, 1982.

SEGALLA, A.L.; OLIVEIRA, H. de; ESPIRONELO, A. \& IGUE, T. Experimentos regionais de variedades de cana-de-açúcar realizados no período de 1970 a 1973. Bragantia, Campinas, 39:115-135, 1980.

TANIMOTO, T. The press method of cane analysis. Hawaiian Planter's Record, Honolulu, 57(2):133-150, 1964. 